\title{
Complete Genome Sequence of Salmonella enterica Serovar Typhimurium Bacteriophage SPN1S
}

\author{
Hakdong Shin, ${ }^{a}$ Ju-Hoon Lee, ${ }^{b}$ Jeong-A Lim, ${ }^{a}$ Hyeryen Kim, ${ }^{a}$ and Sangryeol Ryu ${ }^{a}$ \\ Department of Food and Animal Biotechnology, Department of Agricultural Biotechnology and Center for Agricultural Biomaterials, Seoul National University, Seoul, \\ Korea, ${ }^{a}$ and Department of Food Science and Biotechnology, CHA University, Seongnam, Korea ${ }^{b}$
}

To understand the interaction between the host of pathogenic Salmonella enterica serovar Typhimurium and its bacteriophage, we isolated the bacteriophage SPN1S. It is a lysogenic phage in the Podoviridae family and uses the O-antigen of lipopolysaccharides (LPS) as a host receptor. Comparative genomic analysis of phage SPN1S and the S. enterica serovar Anatum-specific phage $\varepsilon 15$ revealed different host specificities, probably due to the low homology of host specificity-related genes. Here we report the complete circular genome sequence of $S$. Typhimurium-specific bacteriophage SPN1S and show the results of our analysis.

$S^{a}$ almonella infection is one of the most common food-borne illnesses (representing more than $30 \%$ of all bacterial foodborne poisoning) $(4,5)$. More than 1.4 million cases of foodborne Salmonella infection have been reported every year in the United States, and the number of cases has increased by $10 \%$ in recent years $(4,9,13)$. Moreover, the emergence of multidrugresistant Salmonella strains, such as Salmonella enterica serovar Typhimurium DT104, has been getting more problematic $(7,14)$. To control these drug-resistant Salmonella strains, applications of Salmonella-specific bacteriophages have been proposed $(8,15)$. Therefore, it is important to understand the infection mechanism between the Salmonella host and Salmonella-specific phages. To increase our knowledge of this interaction, we isolated $S$. Typhimurium-specific phage SPN1S from environmental water and completely sequenced its genome.

The genomic DNA of phage SPN1S was isolated using an alkaline lysis method (16) and sequenced using Genome Sequencer FLX Titanium (GS-FLX Titanium) technology at Macrogen (Korea) with 130 times coverage. Sequence assembly of quality filtered reads was performed using a 454 Newbler 2.3 assembler. From the complete genome sequence of phage SPN1S, open reading frames (ORFs) were predicted using the GAMOLA automatic annotation program (1) and confirmed using GeneMarkS (3), Glimmer 3.02 (6), and FgenesV (SoftBerry). Conserved protein domain analysis was conducted using BLASTP (2), InterProScan (17), and the NCBI Conserved Domain database (CDD) (12). Prediction of tRNAs was carried out using the tRNAscan-SE program (11).

Bacteriophage SPN1S has a circular genome consisting of 38,684 bp with a GC content of $50.16 \%$ and 52 ORFs but no tRNA. The annotation of this genome revealed genes related to phage packaging (terminase small and large subunits), morphogenesis (a phage head-to-tail connector protein, an endoprotease, a major capsid protein, and a minor structural protein), host specificity (a tail spike protein), conversion of host lipopolysaccharide (LPS) (a GtrA and two copies of lipopolysaccharide modification acyltransferase), host lysis (a holin, an endolysin, an Rz-like protein, and an Rz1), DNA replication/modification (a DNA replication protein, an integrase, an exonuclease VIII/RecE-like protein, and an adenine methylase), and transcription regulation (a transcriptional activator and transcriptional regulators).

Comparative genome analysis of phage SPN1S and S. enterica serovar Anatum-specific phage $\varepsilon 15$ (GenBank accession number
AY150271) revealed that while these two phages are closely related at the DNA level, their host specificity-related genes encoding tail spike/tail fiber proteins are quite different. In addition, the receptor study of phage SPN1S showed that the tail spike protein (SPN1S_0022) interacts with the O-antigen of LPS in S. Typhimurium, suggesting that this phage infects the host strain via LPS as a host receptor (data not shown). Interestingly, Rz1 and Rz-like protein collaborate with the endolysin for host lysis. Expression of the endolysin gene alone using the Escherichia coli gene expression system does not lyse the host strain, but coexpression of the genes encoding endolysin, Rzl, and Rz-like protein does (10). The genome study of phage SPN1S would increase our knowledge of the interaction between the $S$. Typhimurium host and its bacteriophages.

Nucleotide sequence accession number. The complete genome sequence of $S$. Typhimurium phage SPN1S is available in GenBank under accession number JN391180.

\section{ACKNOWLEDGMENTS}

This work was supported by a National Research Foundation of Korea (NRF) grant (20090078983) funded by the Ministry of Education, Science and Technology. H. Shin and J.-A. Lim were recipients of graduate fellowships provided by the Ministry of Education, Science and Technology through the Brain Korea 21 Project.

\section{REFERENCES}

1. Altermann E, Klaenhammer TR. 2003. GAMOLA: a new local solution for sequence annotation and analyzing draft and finished prokaryotic genomes. OMICS 7:161-169.

2. Altschul SF, Gish W, Miller W, Myers EW, Lipman DJ. 1990. Basic local alignment search tool. J. Mol. Biol. 215:403-410.

3. Besemer J, Lomsadze A, Borodovsky M. 2001. GeneMarkS: a selftraining method for prediction of gene starts in microbial genomes. Implications for finding sequence motifs in regulatory regions. Nucleic Acids Res. 29:2607-2618.

4. Centers for Disease Control and Prevention. 2007. Bacterial foodborne and diarrheal disease national case surveillance. Annual report, 2005. US

Received 31 October 2011 Accepted 1 November 2011

Address correspondence to Sangryeol Ryu, sangryu@snu.ac.kr.

H.S. and J.-H.L. contributed equally to this work.

Copyright $\odot$ 2012, American Society for Microbiology. All Rights Reserved.

doi:10.1128/JVI.06696-11 
Department of Health and Human Services, Centers for Disease Control and Prevention, Atlanta, GA.

5. Centers for Disease Control and Prevention. 2008. Salmonella surveillance. Annual summary, 2006. US Department of Health and Human Services, Centers for Disease Control and Prevention, Atlanta, GA.

6. Delcher AL, Bratke KA, Powers EC, Salzberg SL. 2007. Identifying bacterial genes and endosymbiont DNA with Glimmer. Bioinformatics 23:673-679.

7. Fluit AC. 2005. Towards more virulent and antibiotic-resistant Salmonella? FEMS Immunol. Med. Microbiol. 43:1-11.

8. GarcíA P, Martínez B, Obeso JM, Rodríguez A. 2008. Bacteriophages and their application in food safety. Lett. Appl. Microbiol. 47:479-485.

9. Gilliss D, et al. 2011. Vital signs: incidence and trends of infection with pathogens transmitted commonly through food-foodborne diseases active surveillance network, 10 U.S. sites, 1996-2010. MMWR Morb. Mortal. Wkly. Rep. 60:749-755.

10. Krupovic M, Cvirkaite-Krupovic V, Bamford DH. 2008. Identification and functional analysis of the Rz/Rzl-like accessory lysis genes in the membrane-containing bacteriophage PRD1. Mol. Microbiol. 68:492-503.
11. Lowe TM, Eddy SR. 1997. tRNAscan-SE: a program for improved detection of transfer RNA genes in genomic sequence. Nucleic Acids Res. 25: 955-964.

12. Marchler-Bauer A, et al. 2007. CDD: a conserved domain database for interactive domain family analysis. Nucleic Acids Res. 35:D237-D240.

13. Mead PS, et al. 1999. Food-related illness and death in the United States. Emerg. Infect. Dis. 5:607-625.

14. Meunier D, et al. 2002. Salmonella enterica serotype Typhimurium DT 104 antibiotic resistance genomic island I in serotype Paratyphi B. Emerg. Infect. Dis. 8:430-433.

15. O'Flaherty S, Ross RP, Coffey A. 2009. Bacteriophage and their lysins for elimination of infectious bacteria. FEMS Microbiol. Rev. 33: 801-819.

16. Wilcox SA, Toder R, Foster JW. 1996. Rapid isolation of recombinant lambda phage DNA for use in fluorescence in situ hybridization. Chromosome Res. 4:397-398.

17. Zdobnov EM, Apweiler R. 2001. InterProScan—an integration platform for the signature-recognition methods in InterPro. Bioinformatics 17: $847-848$. 